\title{
Identification of two novel Duchenne muscular dystrophies mutations in patients with Becker muscular dystrophy
}

Dahye Kim¹, Yoon-Myung Kim¹, Go Hun Seo', Gu Hwan Kim², Han Wook Yoo ${ }^{1,2}$, Mi-Sun Yum¹, Tae-Sung Ko', and Beom Hee Lee ${ }^{1,2, *}$ 'Department of Pediatrics, Asan Medical Center Children's Hospital, University of Ulsan College of Medicine, Seoul, Korea ${ }^{2}$ Medical Genetics Center, Asan Medical Center Children's Hospital, University of Ulsan College of Medicine, Seoul, Korea

Duchenne and Becker muscular dystrophies (DMD and BMD, respectively) are X-linked neuromuscular disorders characterized by progressive muscle weakness and severe skeletal muscle degeneration. BMD is a milder form with a later onset. Patients with $\mathrm{BMD}$ tend to survive much longer than those with $\mathrm{DMD}$. The differentiation between $\mathrm{DMD}$ and $\mathrm{BMD}$ is important in the genetic counseling of affected patients and their families. Since muscle biopsies are invasive procedures, the differential diagnosis of $\mathrm{BMD}$ and $\mathrm{DMD}$ is often dependent on the mutation identified in the $D M D$ gene in affected patients. However, when a novel $D M D$ mutation is identified, the differential diagnosis should be based on muscle biopsy findings with other clinical findings. Here we describe two Korean patients with BMD confirmed by muscle biopsy and genetic testing. Two novel exonic deletions in the $D M D$ gene were identified.

Key words: Becker muscular dystrophy, Duchenne muscular dystrophy, Mutation.

\section{Introduction}

Duchenne and Becker muscular dystrophies (DMD, OMIM \#310200 and BMD, OMIM \#300376; respectively) are caused by mutations in $D M D$, the dystrophin-coding gene. These conditions are inherited in an $\mathrm{X}$-linked recessive manner with a prevalence of 1 in 4,000 to 5,000 new born males [1].

DMD and BMD are characterized by progressive muscular weakness. DMD is a severely disabling neuromuscular disease that leads to premature death of patients at approximately 19 years of age due to cardiac or respiratory failure. In contrast, BMD is a milder form with a later onset and slower rate of progression, and patients older than 16 years remain ambulatory and may have near normal lives [2,3].

The differential diagnosis of BMD and DMD in suspected cases is based on their clinical findings, muscle biopsy, and the mutations in DMD. Among these, positive staining for dystrophin in muscle biopsies is an important finding in the differential diagnosis $[4,5]$. However, since muscle biopsies are invasive procedures, the differential diagnosis of BMD and DMD is often dependent on the mutations in DMD gene identified in the patient. According to the mutation database or previous reports, each DMD mutation is classified as either a DMD- or BMDcausing mutation [6]. However, when a novel DMD mutation is identified, the differential diagnosis should be based on the muscle biopsy findings along with other clinical findings.

\footnotetext{
Received: 17 July 2017, Revised: 6 October 2017, Accepted: 13 October 2017, Published: 31 December 2017

*Corresponding author: Beom Hee Lee, M.D., Ph.D.

Department of Pediatrics, Asan Medical Center Children's Hospital, University of Ulsan College of Medicine, 88 Olympic-ro 43-gil, Songpa-gu, Seoul 05505, Korea.

Tel: +82-2-3010-5950, Fax: +82-2-473-3725, E-mail: bhlee@amc.seoul.kr

Conflict of interest: The authors declare that they do not have any conflicts of interest.

(c) This is an open-access article distributed under the terms of the Creative Commons Attribution Non-Commercial License (http://creativecommons.org/licenses/by-nc/4.0/) which permits unrestricted non-commercial use, distribution, and reproduction in any medium, provided the original work is properly cited.

(c) Copyright 2017 by the Korean Society of Medical Genetics and Genomics www.e-kjgm.org
} 
Herein, we report two novel $D M D$ mutations identified in suspected patients as having DMD or BMD. Based on the clinical and histological findings, the confirmatory diagnosis was BMD in both cases.

\section{Case}

\section{Case 1}

A Korean male patient was born after 40 weeks of gestation with a birth weight of 3,400 g. Pregnancy, labor, and vaginal delivery were uneventful. His physical growth and verbal, motor, and cognitive developments were within normal ranges.

A myopathy of mild severity was noted, when he was 9 years old. His serum creatine kinase (CK) level was 9,287 IU/L (normal range, $<150 \mathrm{IU} / \mathrm{L}$ ). Mild muscle weakness was noted in the lower extremities (muscle power of grade 5-) with waddling gaits, minimal Gowers' sign, and mild calf muscle pseudohypertrophy. The patient also had no family history of muscular diseases.

The result of pediatric echocardiography was normal cardiac function and structure; the left ventricular ejection fraction (LVEF) was 66.09\% (normal range, >55\%) and the left ventricular fractional shortening (LVFS) was 35.96\% (normal range, $>25 \%$ ).

Genomic DNA was isolated from the patient's peripheral leukocytes. Multiple ligation-dependent probe amplifications (MLPAs) were performed for the detection of deletions and duplications in DMD. The DMD MLPA probemix P034 and P035 (MRCHolland, Amsterdam, The Netherlands) was used according to the manufacturer's instructions. The products were separated by electrophoresis on an ABI3130xI genetic analyzer (Applied
Biosystems, Foster City, CA, USA). The peak height of the probes was analyzed using GeneMarker software (SoftGenetics, State College, PA, USA). The probes were normalized and the ratios computed. MLPA analysis identified a deletion of exons 78 and 79 in $D M D$, which has not been previously reported (Fig. 1).

Muscle biopsy was performed in his vastus lateralis muscle, and histologic evaluations via light microscopy revealed mild variations of myofibers. Few necrotic and regenerating fibers, as well as patchy positive staining pattern for dystrophin were observed by immunohistochemistry. In addition, few atrophic fibers with non-specific degeneration were observed by electron microscopy (Fig. 2).

Oral deflazacort ( $1 \mathrm{mg} / \mathrm{kg} /$ day) was administered since 9 years of age. During 12 months of treatment, deflazacort was tapered gradually, and his general condition has been stable. Though the clinical symptoms of the patient were well controlled, the CK level was $13,145 \mathrm{IU} / \mathrm{L}$ at the latest follow-up at 10 years of age, which was similar to his initial level.

\section{Case 2}

The Korean male was born after 40 weeks of gestation with a birth weight 3,200 g. Pregnancy, labor, and vaginal delivery were uneventful. Growth and development had been normal. This patient at 11 years of age, had no limitation to daily activities, but increased serum CK levels $(6,295 \mathrm{IU} / \mathrm{L})$ were found when he visited the hospital with his flank pain. Clinical examination revealed normal muscle power in the extremities with minimal calf muscle pseudohypertrophy. Gowers' sign was negative, and there was no family history of muscle disease.

Echocardiography was performed on the patient, revealing

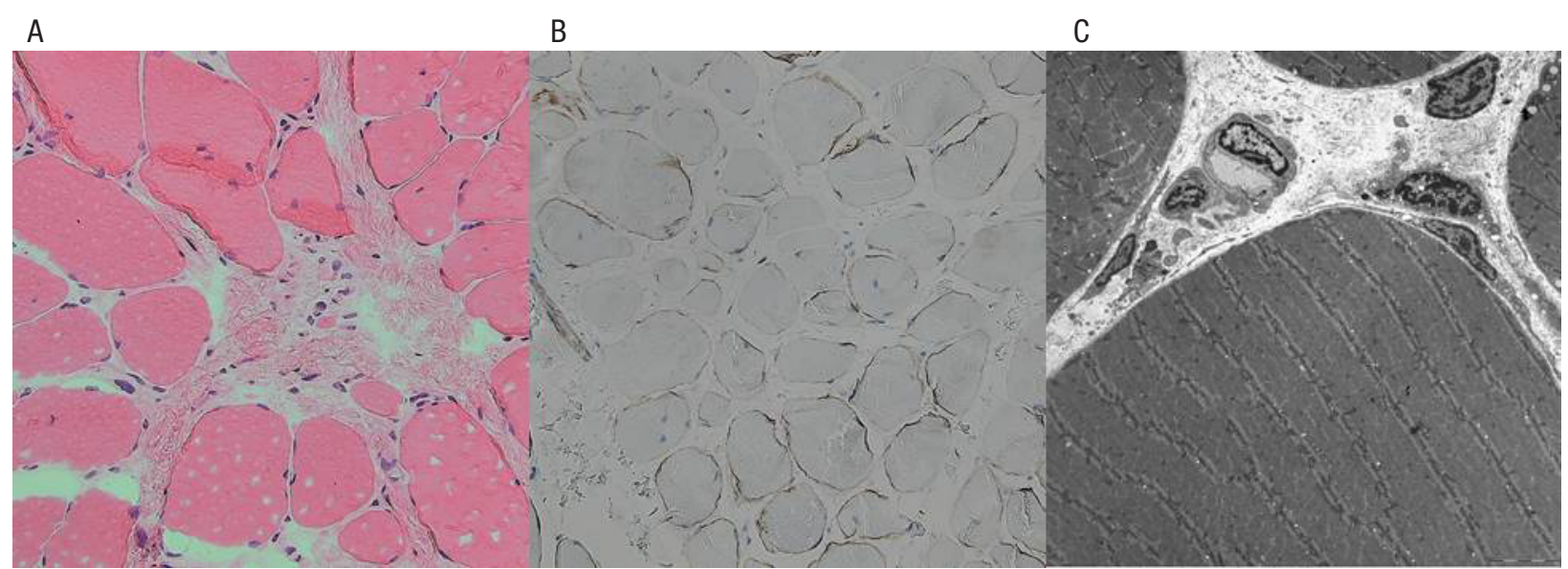

Fig. 1. (A) A mild variation of myofibers and few necrotic, regenerating fibers were observed in light microscopic examination, light microscopy (H\&E stain, $\times 200$ ). (B) Patchy positive staining was revealed for dystrophin in the immunohistochemistry, light microscopy (dystrophin stain, $\times 2,000$ ). (C) Few atrophic fibers with non-specific degeneration change in electron microscopic examination, $\times 2,500$. 
MLPA analysis of DMD gene. (P034-A2; P035-A2, MRC Holland)

DMD exon1-10, 21-30,41-50,61-70 set

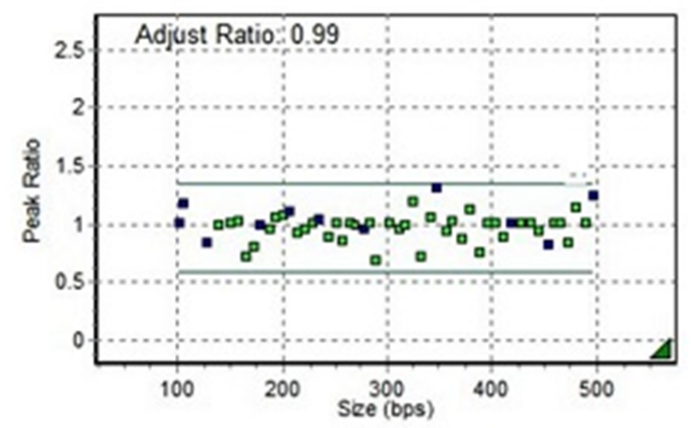

c.11015-?_11058+?del(exon78-79deletion)
DMD exon11-20,31-40,51-60,71-79 set

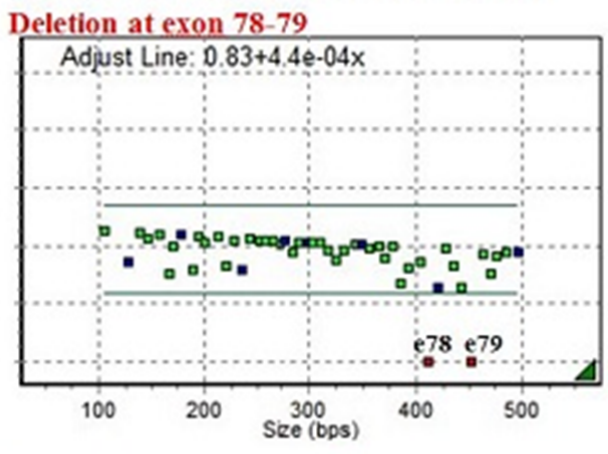

Analyzed by GeneMarker v.1.7

Fig. 2. Multiple ligation-dependent probe amplification (MLPA) analysis of patient 1. Deletion of exon 78 and 79 of the DMD gene was identified.

A

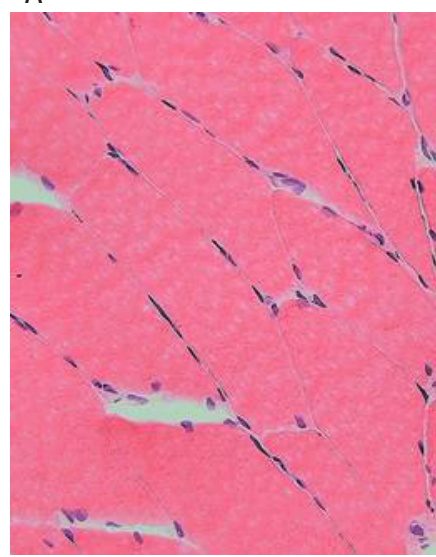

B

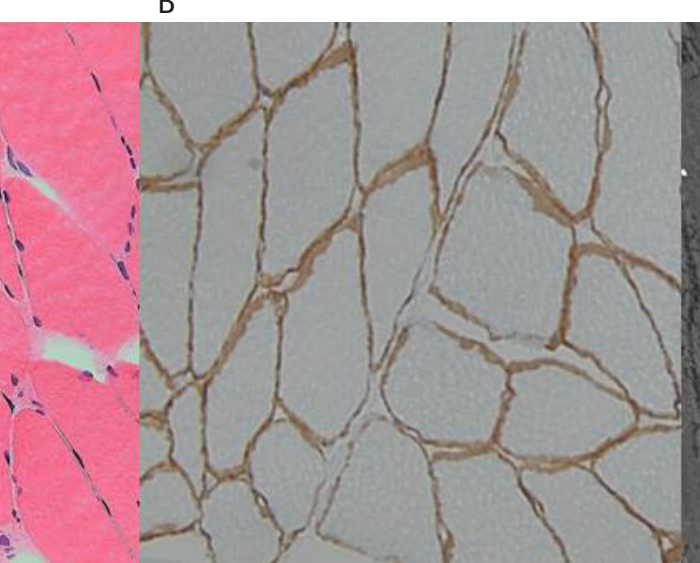

C

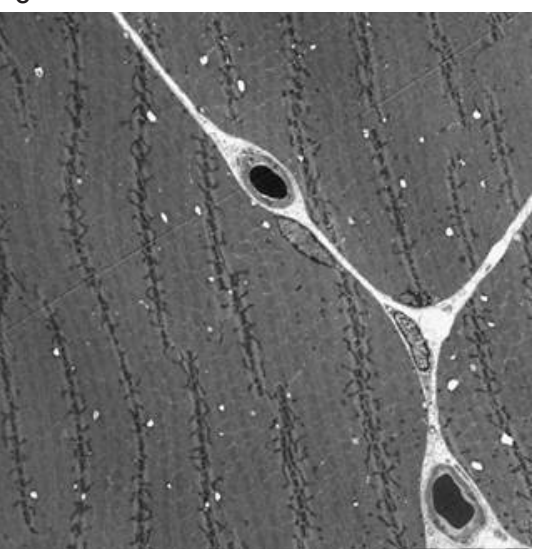

Fig. 3. (A) No diagnostic abnormalities in light microscopic examination, light microscopy (H\&E stain, $\times 2,000$ ). (B) Dystrophin presents near the surface of muscle fibers in immunohistochemistry, light microscopy (dystrophin stain, $\times 200$ ). (C) Normal findings in electron microscopy, $\times 2,000$.

good ventricular function and normal structure of heart; the LVEF was $64.99 \%$ and the LVFS was $34.37 \%$.

Genomic DNA was extracted from the patient's peripheral leukocytes, and MLPA analysis detected a deletion of exons 30-38 in DMD, which has not been previously reported. MLPA analysis of his mother and elder sister revealed heterozygous deletions of exons 30-38 in DMD. The deletions were identical to that detected in the patient; thus they were confirmed as carriers (Fig. 3).

A muscle biopsy was performed in his vastus lateralis muscle, and immunohistochemical staining via light microscopy identified unremarkable histopathologic findings with diffusely stained dystrophin. Furthermore, electron microscopy revealed normal findings (Fig. 4).

The patient was treated with oral deflazacort at starting doses of $1 \mathrm{mg} / \mathrm{kg} /$ day since 11 years of age. The dosage of deflazacort was slowly tapered for 15 months.

The CK level of the patient remarkably decreased to $286 \mathrm{IU} / \mathrm{L}$ at the latest follow-up at 12 years of age. His clinical sign was equal to pretreatment status as intact motor power with mild hypertrophy of calf muscle.

\section{Discussion}

DMD is a severe childhood neuromuscular disorder characterized by progressive dystrophy of the skeletal muscles. DMD occurs in early childhood, resulting in difficulties in physical exercise [2]. In addition, patients with DMD have a high rate of cognitive impairment and learning disabilities, which are related to the disruption of dystrophin isoforms in the central nervous 


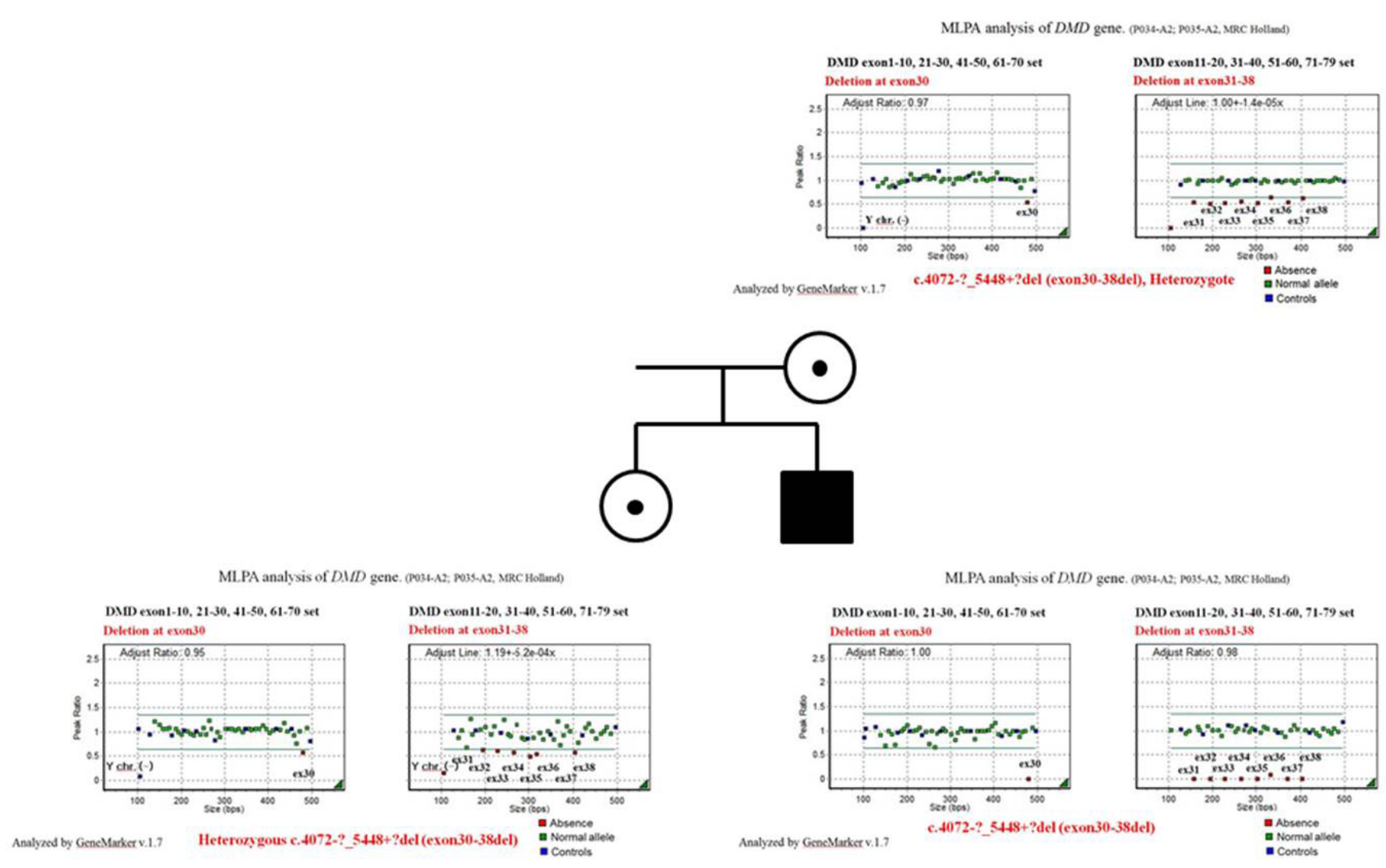

Fig. 4. Deletion of exon 30-38 deletions of the DMD gene was identified in patient 2. Heterozygous deletion of exon 30-38 of the DMD gene was checked in mother and sister of patient 2.

system [7].

In BMD, muscle wasting and weakness are also noted as in DMD, but BMD is a milder form with a later onset, and patients have a much longer survival in comparison to DMD. However, the severities were different among patients with BMD as described in our study. Some of the patients with BMD are asymptomatic while others become wheelchair-bound around 16 years of age. The patients could survive until very old ages or some of them could die from an early heart failure $[8,9]$. Although the underlying mechanism of this variability is not fully understood, it is likely that both levels and functionality of the internally deleted dystrophins play a significant role [10]. Anthony et al. [11] suggested that the patients with BMD who have milder phenotypes have higher expression levels of dystrophin. On the other hand, recent study showed that there was no linear correlation between dystrophin levels and disease severity in patients with BMD [12]. If the dystrophin levels are greater than the threshold level (about 30\%), then the structural characteristics of the internally deleted dystrophins, rather than the quantity of the dystrophin, could affect the phenotype [13]. Therefore, the structure modifications of dystrophin determined by the site of deletion in addition to its expression level modulate the severity of BMD [10].

In the current study, we described the general clinical characteristics, genetic findings, and results of muscle biopsies of two Korean patients with BMD. Consistent with previous observations, our patients showed clinical features of commonly observed in patients with BMD but with different severity. One patient had mild muscle weakness, minimal Gowers' sign, and calf muscle pseudohypertrophy, whereas the other patient only had mild calf muscle enlargement and had been nearly asymptomatic. Elevations of CK levels were similar between the two patients, and cardiac function was normal. Two different novel exonic deletions of $D M D$ were identified; a deletion of exons 78 and 79 in DMD in one patient and a deletion of exons 30-38 in the mildly-affected patient. Of note, the muscle biopsy results are different between the two patients, one had few degeneration changes and the other had normal histopathologic features. We could assume that two patients were BMD, since the total absence of dystrophin protein in immunocytochemistry indicates DMD, and the partial lack of dystrophin protein means BMD $[2,4]$. The expression pattern of dystrophin was abundant 
in the patient with the deletion in exons 30-38 in contrast to a patchy intermittent expression pattern in the patient with the deletion in exons 78 and 79. Thus, it is expected that dystrophin lacking exons 30-38 is more structurally stable than dystrophin lacking exons 78 and 79, which is associated with the milder BMD phenotype.

Recently, advances have been noted in the treatment of DMD by the development of exon skipping therapies [14]. The skipping of one or more exons flanking the specific deletion site in patients with DMD allows for the introduction of BMD-like dystrophin and as a result, converts severe DMD into a BMDtype disease $[15,16]$. In this regard, gathering clinical and genetic information of patients with BMD has become more important for determining a precise genotype-phenotype correlation [14]. Because the phenotypic severity is different according to the deletion site in patients with BMD, exon-skipping therapies would also be affected by the specific site to be skipped.

In conclusion, it is important to report novel $D M D$ gene mutations for the diagnosis of BMD and treatment of DMD. Additionally, novel $D M D$ gene mutations need to be confirmed by muscle biopsy findings, especially since the expression pattern of dystrophin appears to be associated with the phenotypic severity among patients with BMD.

\section{Acknowledgements}

This study was supported in part by a grant from the National Research Foundation of Korea, funded by the Ministry of Education, Science, and Technology (NRF-2016M3A9B4915706).

\section{References}

1. Mah JK, Korngut L, Dykeman J, Day L, Pringsheim T, Jette N. A systematic review and meta-analysis on the epidemiology of Duchenne and Becker muscular dystrophy. Neuromuscul Disord 2014;24:48291.

2. Bushby K, Finkel R, Birnkrant DJ, Case LE, Clemens PR, Cripe $L$, et al. Diagnosis and management of Duchenne muscular dystrophy, part 1: diagnosis, and pharmacological and psychosocial management. Lancet Neurol 2010;9:77-93.

3. Bushby KM, Gardner-Medwin D, Nicholson LV, Johnson MA, Haggerty ID, Cleghorn NJ, et al. The clinical, genetic and dystrophin characteristics of Becker muscular dystrophy. II. Correlation of phenotype with genetic and protein abnormalities. J Neurol 1993;240:105-12.

4. Hoffman EP, Fischbeck KH, Brown RH, Johnson M, Medori R, Loike
JD, et al. Characterization of dystrophin in muscle-biopsy specimens from patients with Duchenne's or Becker's muscular dystrophy. $\mathrm{N}$ Engl J Med 1988;318:1363-8.

5. Nicholson LV, Johnson MA, Bushby KM, Gardner-Medwin D, Curtis A, Ginjaar IB, et al. Integrated study of 100 patients with Xp21 linked muscular dystrophy using clinical, genetic, immunochemical, and histopathological data. Part 3. Differential diagnosis and prognosis. J Med Genet 1993;30:745-51.

6. Bushby KM. Genetic and clinical correlations of Xp21 muscular dystrophy. J Inherit Metab Dis 1992;15:551-64.

7. Banihani R, Smile S, Yoon G, Dupuis A, Mosleh M, Snider A, et al. Cognitive and neurobehavioral profile in boys with Duchenne muscular dystrophy. J Child Neurol 2015;30:1472-82.

8. Takeshima Y, Yagi M, Okizuka Y, Awano H, Zhang Z, Yamauchi Y, et al. Mutation spectrum of the dystrophin gene in 442 Duchenne/Becker muscular dystrophy cases from one Japanese referral center. J Hum Genet 2010;55:379-88.

9. Yazaki M, Yoshida K, Nakamura A, Koyama J, Nanba T, Ohori N, et al. Clinical characteristics of aged Becker muscular dystrophy patients with onset after 30 years. Eur Neurol 1999;42:145-9.

10. Nicolas $A$, Raguénès-Nicol $C$, Ben Yaou $R$, Ameziane-Le Hir $S$, Chéron $A$, Vié $V$, et al. Becker muscular dystrophy severity is linked to the structure of dystrophin. Hum Mol Genet 2015;24:1267-79.

11. Anthony K, Cirak S, Torelli S, Tasca G, Feng L, Arechavala-Gomeza V, et al. Dystrophin quantification and clinical correlations in Becker muscular dystrophy: implications for clinical trials. Brain 2011;134:354759.

12. van den Bergen JC, Wokke BH, Janson AA, van Duinen $S G$, Hulsker MA, Ginjaar HB, et al. Dystrophin levels and clinical severity in Becker muscular dystrophy patients. J Neurol Neurosurg Psychiatry 2014;85:747-53.

13. Neri M, Torelli S, Brown $S$, Ugo I, Sabatelli P, Merlini L, et al. Dystrophin levels as low as 30\% are sufficient to avoid muscular dystrophy in the human. Neuromuscul Disord 2007;17:913-8.

14. Yang J, Li SY, Li YQ, Cao JQ, Feng SW, Wang YY, et al. MLPA-based genotype-phenotype analysis in 1053 Chinese patients with DMD/ BMD. BMC Med Genet 2013;14:29.

15. Barzegar M, Habibi P, Bonyady M, Topchizadeh V, Shiva S. Exon deletion pattern in duchene muscular dystrophy in north west of Iran. Iran J Child Neurol 2015;9:42-8.

16. Cirak S, Arechavala-Gomeza V, Guglieri M, Feng L, Torelli S, Anthony $K_{1}$ et al. Exon skipping and dystrophin restoration in patients with Duchenne muscular dystrophy after systemic phosphorodiamidate morpholino oligomer treatment: an open-label, phase 2, dose-escalation study. Lancet 2011;378:595-605. 